\title{
Can integrating the Memory Support Intervention into cognitive therapy improve depression outcome? Study protocol for a randomized controlled trial
}

Allison G. Harvey ${ }^{1 *}$, Lu Dong ${ }^{1}$, Jason Y. Lee ${ }^{1}$, Nicole B. Gumport ${ }^{1}$, Steven D. Hollon², Sophia Rabe-Hesketh', Kerrie Hein ${ }^{1}$, Kirsten Haman², Mary E. McNamara', Claire Weaver ${ }^{1}$, Armando Martinez' ${ }^{1}$ Haruka Notsu', Garret Zieve ${ }^{1}$ and Courtney C. Armstrong ${ }^{1}$

\begin{abstract}
Background: The Memory Support Intervention was developed in response to evidence showing that: (1) patient memory for treatment is poor, (2) poor memory for treatment is associated with poorer adherence and poorer outcome, (3) the impact of memory impairment can be minimized by the use of memory support strategies and (4) improved memory for treatment improves outcome. The aim of this study protocol is to conduct a confirmatory efficacy trial to test whether the Memory Support Intervention improves illness course and functional outcomes. As a "platform" for the next step in investigating this approach, we focus on major depressive disorder (MDD) and cognitive therapy (CT).

Method/design: Adults with MDD ( $n=178$, including $20 \%$ for potential attrition) will be randomly allocated to CT + Memory Support or CT-as-usual and will be assessed at baseline, post treatment and at 6 and 12 months' follow-up (6FU and 12FU). We will compare the effects of CT + Memory Support vs. CT-as-usual to determine if the new intervention improves the course of illness and reduces functional impairment (aim 1). We will determine if patient memory for treatment mediates the relationship between treatment condition and outcome (aim 2). We will evaluate if previously reported poor treatment response subgroups moderate target engagement (aim 3).

Discussion: The Memory Support Intervention has been developed to be "transdiagnostic" (relevant to a broad range of mental disorders) and "pantreatment" (relevant to a broad range of types of treatment). This study protocol describes a "next step" in the treatment development process by testing the Memory Support Intervention for major depressive disorder (MDD) and cognitive therapy (CT). If the results are promising, future directions will test the applicability to other kinds of interventions and disorders and in other settings.
\end{abstract}

Trial registration: ClinicalTrials.gov, ID: NCT01790919. Registered on 6 October 2016.

Keywords: Memory support, Depression, Cognitive therapy, Transdiagnostic, Experimental therapeutics

\footnotetext{
* Correspondence: aharvey@berkeley.edu

'Department of Psychology, University of California, Berkeley, 3210 Tolman

Hall \#1650, Berkeley, CA 94720-1650, USA

Full list of author information is available at the end of the article
} 


\section{Background}

Patients accurately recall only about one third of the recommendations made during a physician visit [1-8] and during a cognitive behavior therapy (CBT) session $[9,10]$. In one study, $25 \%$ of patients remembered recommendations that were not made [1]. Recall is particularly poor for advice for healthbehavior change [11]. Perhaps not surprisingly, poor memory for the content of treatment is associated with poor adherence to medical treatments for chronic conditions [12-16], which is known to be associated with worse outcome [17]. Also, poor memory for CBT is associated with poorer outcome [10].

We have offered various explanations for these findings [18]. First, even when memory is functioning optimally, fallibility is possible at initial encoding, storage or retrieval [19]. Second, a CBT treatment session is typically $50 \mathrm{~min}$ long, covers complex information, and can elicit negative emotion. Negative emotion is associated with attentional biasing and narrowing, which impacts encoding [20]. Third, the odds are stacked against people learning, generalizing and transferring knowledge to new situations; this is known as the transfer of learning problem [21, 22]. Fourth, memory deficits and biases are common across mental disorders [23-27] and memory deficits are associated with poorer memory for treatment [28, 29].

The good news is that the impact of memory impairment can be minimized. Memory encoding and retention can be markedly improved by the use of memory support techniques. This has been demonstrated in medical visits [13, 30], and among older adults [31] as well as for people with memory impairments associated with Alzheimer's disease, vascular dementia [32] and frontal lobe dysfunction [33].

We developed and tested the Memory Support Intervention, which is designed to improve patient memory for treatment. This approach is not intended to have a direct effect on improving memory functioning per se. The Memory Support Intervention was distilled from the cognitive science and education literature based on carefully honed criteria [18]. A small underpowered trial has provided preliminary evidence that the intervention exerts a measurable effect on patient memory for treatment and demonstrates a clinical effect [34].

The Memory Support Intervention has been developed to be "transdiagnostic" (relevant to a broad range of mental disorders) and "pantreatment" (relevant to a broad range of types of treatment). However, investigating these possible broader applications seems premature without first establishing efficacy, which is the aim of the study described in this paper. To create a platform for the "next step" in investigating the approach, we will recruit patients who meet diagnostic criteria for major depressive disorder (MDD) and focus on one biopsychosocial intervention-cognitive therapy (CT). If the results are promising, future directions will test the applicability to other kinds of interventions and disorders and in other settings.

Why focus on MDD? First, MDD is prevalent and causes impairment $[35,36]$; second, there is a need to improve treatments for MDD because a proportion of patients do not recover and of those who do recover, the majority relapse [37]; third, memory is poor in MDD and is modifiable [38, 39]; fourth, preliminary data suggest that improving memory for treatment in MDD improves outcome [34, 40, 41].

Why focus on CT for MDD? CT for MDD is well articulated and has been widely studied. Meta-analyses confirm CT as a frontline treatment $[42,43]$. Despite these impressive outcomes, there is room for improvement $[44,45]$.

This study protocol addresses two additional considerations. First, older age, lower intelligence and chronic depression each predict poorer response to treatment for depression, including CT [46]. Impairment in declarative memory is also associated with poorer outcome [47-50]. Moreover, fewer years of education has been associated with a positive response to CT + Memory Support relative to more years of education [34]. We hypothesize that all of these poorer response groups may derive special benefit from the Memory Support Intervention. Hence, while we expect all patients to benefit from the Memory Support Intervention, we have planned analyses to determine if these subgroups stand to gain the greatest advantage from experimental facilitation of memory for treatment. Second, there is evidence that improved mood in MDD is associated with improved declarative memory [51], although remitted MDD patients still exhibit significant memory deficits [23]. We have planned analyses to address this potential confound.

Over a 4-year period, adults with MDD $(n=178$, including $20 \%$ for attrition) will be randomly allocated to CT + Memory Support or CT-as-usual and will be assessed at baseline, post treatment and at 6 and 12 months' follow-up (6FU and 12FU). The first aim is to assess whether the Memory Support Intervention (1) improves the course of illness and (2) reduces functional impairment. Compared to those receiving CT-as-usual, we expect that patients receiving $\mathrm{CT}+$ Memory Support will experience greater symptom reduction on measures of course of illness, as defined by the American College of Neuropsychopharmacology (ACNP) Task Force criteria, and on measures of functional impairment. The second aim is to evaluate whether patient memory for treatment mediates the relationship between treatment condition and outcome. Relative to CT-as-usual, we expect CT + Memory Support to be associated with better treatment outcome, and will be mediated by better patient memory for treatment, measured by the Patient Treatment Recall 
Task. The third aim is to evaluate if previously reported poor treatment response subgroups moderate target engagement. We expect that the relationship between memory support dose and outcome will be positive and stronger among those who are older, have lower IQ, have more chronic depression, have poorer baseline declarative memory performance and have fewer years of education.

\section{Method/design}

\section{Study design and setting}

This is a prospective randomized controlled study. Adults $(n=178)$ who meet criteria for MDD will be randomly assigned, in a 1:1 parallel group design, to CT + Memory Support or CT-as-usual (see Fig. 1 for a flow chart of the study design). Randomization is stratified by age $(\leq 49,50+$ years) and depression chronicity ( $<2$ years, $\geq 2$ years) [46]. Participants receive a battery of outcome measures pre-treatment and post-treatment (i.e., within 2 weeks after the final treatment session) and at 6 and 12 months' follow-up. Additional assessments of patient and therapist memory for treatment take place in weeks $4,8,12$ and 16 of treatment for the mediation analysis.

The assessment team is blind to treatment allocation. Randomization will be conducted using a computerized, random-number generator where the planned stratified randomization is a part of the generation of the allocation sequence. Only the project coordinators in charge of randomization and of the Memory Support Rating Scale (MSRS) scoring know the treatment allocation of each participant. A Data Safety and Monitoring Board (DSMB) will review the study every 6 months during the active treatment phase. The Standard Protocol Items: Recommendations for Interventional Trials (SPIRIT 2013) Checklist (see Additional file 1) and Figure (see Fig. 2 Standard Protocol Item: Recommendation for Interventional Trials diagram) are provided [52].

For participants who discontinue, the assessment team will endeavor to collect all assessment data, prioritizing the primary outcomes.

\section{Participants}

A total of 178 adults who meet the criteria will be recruited. Recruitment will be conducted in the Bay Area, CA, United States by clinician referral and advertisements. Eligibility is assessed first by a phone interview and then, if the individual is eligible after the phone interview, a more detailed, in-person interview. To enhance representativeness and generalizability, the inclusion and exclusion criteria are kept to a minimum.

\section{Inclusion criteria}

The inclusion criteria are:
- Age $18+$ years

- Willing and able to give consent. Participants must consent to being video-recorded (necessary for MSRS scoring) and to NIMH data sharing ${ }^{1}$

- English language fluency

- Diagnosis of major depressive disorder (MDD), first episode, recurrent or chronic according to the Diagnostic and Statistical Manual of Mental Disorders, Fifth Edition (DSM-5)

- Minimum score 26 or above on the Inventory of Depressive Symptomatology, Self-Report (IDS-SR). This cutoff denotes at least "moderate" depression

- If taking medications for mood, medications must be stable for the past 4 weeks

\section{Exclusion criteria}

The exclusion criteria are:

- History of bipolar disorder

- History of psychosis or psychotic features

- Lifetime history of failure to respond to four or more sessions of CBT/CT for depression

- Current non-psychotic disorder if it constitutes the principal diagnosis and if it requires treatment other than that offered in the project. "Principal" is defined as the disorder currently most distressing and disabling, using a widely accepted severity rating scale capturing distress and interference $(0-8,4+$ indicates clinical severity)

- Moderate or severe substance use in the past 6 months where "moderate" is defined as 4-5 symptoms and "severe" is defined as $6+$ symptoms of those listed in DSM-5 for each of the substancerelated disorders

- Evidence of any medical disorder or condition that could cause depression, preclude participation in $\mathrm{CT}$, or is associated with memory problems, that is not currently stabilized and/or managed under the care of a physician or the presence of an active and progressive physical illness or neurological degenerative disease

- Current suicide risk sufficient to preclude treatment on an outpatient basis (assessed by the ColumbiaSuicide Severity Rating Scale) or current homicide risk (assessed by our staff or referring treatment provider)

- Pregnancy or breastfeeding

- Not able/willing to participate in and/or complete the pre-treatment assessments

Excluding participants whose medications need to be changed is neither feasible nor representative of clinical practice [53]. Strategies to manage potential medication confounds are: (1) medications must be at 


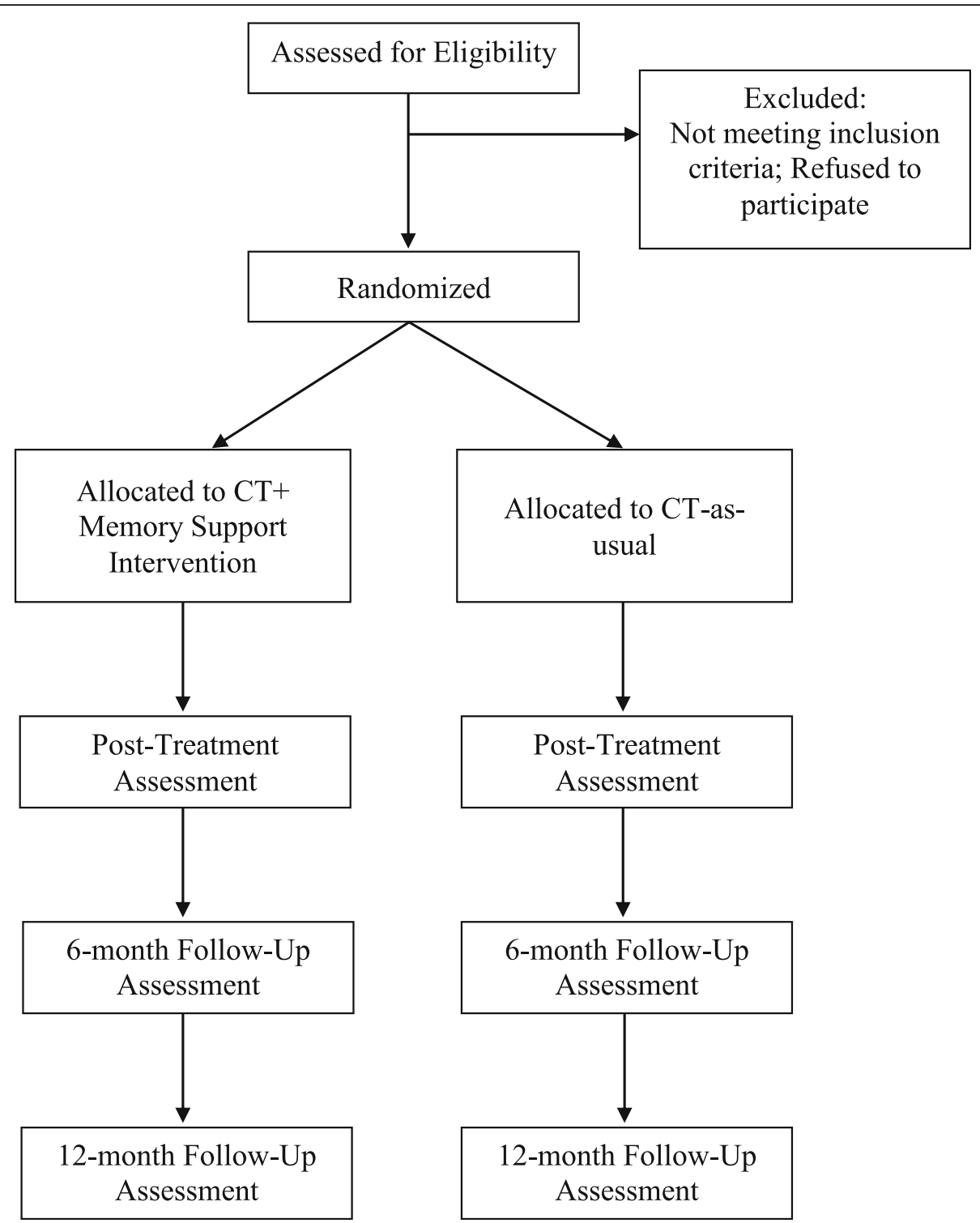

Fig. 1 Standard Protocol Items: Anticipated patient flow for the randomized clinical trial

stable doses for 4 weeks prior to randomization and (2) medication use and changes, along with participation in other treatments/therapy, will be recorded. All medication decisions will ultimately rest with the treating physician and participant.

Following the precedent set in prior research [54], participants with a lifetime history of failure to respond to four or more sessions of CBT for depression will be excluded. Participants who have engaged in moderate or severe substance use in the past 6 months will also be excluded. "Moderate" substance use is defined as four to five symptoms and "severe" substance use is defined as six or more of the symptoms listed in the DSM-5 for each of the substance-related disorders.

\section{Measures}

The primary and secondary outcomes are presented in Additional file 2. Additional file 3 lists all of the measures as well as the timing for administration. Except where specified in Additional file 3, the measures described below will be delivered at each assessment point.

In addition to demographics (age, contact information, gender, race/ethnicity, family, education, employment, living arrangements, government assistance, housing) and assessment of medical history the following measures will be administered:

\section{Diagnostic}

To evaluate current and past psychiatric disorders the Structured Clinical Interview for DSM-5 (SCID) $[55,56]$ 


\begin{tabular}{|c|c|c|c|c|c|c|c|}
\hline \multirow[b]{3}{*}{ TIMEPOINT } & \multicolumn{7}{|c|}{ STUDY PERIOD } \\
\hline & \multirow[b]{2}{*}{ Enrollment } & \multirow[b]{2}{*}{$\begin{array}{c}\text { Pre- } \\
\text { Treatment } \\
\text { Assessment }\end{array}$} & \multirow[b]{2}{*}{ Allocation } & \multicolumn{4}{|c|}{ Post-Allocation } \\
\hline & & & & Intervention & $\begin{array}{c}\text { Post- } \\
\text { Treatment } \\
\text { Assessment }\end{array}$ & $\begin{array}{l}6- \\
\text { Month } \\
\text { Follow- } \\
\text { Up }\end{array}$ & $\begin{array}{l}12- \\
\text { Month } \\
\text { Follow- } \\
\text { Up }\end{array}$ \\
\hline \multirow{4}{*}{$\begin{array}{r}\text { ENROLMENT: } \\
\text { Eligibility Screen } \\
\text { Informed Consent } \\
\text { Allocation }\end{array}$} & & & & & & & \\
\hline & $\mathrm{X}$ & & & & & & \\
\hline & $X$ & $X$ & & & & & \\
\hline & & & $x$ & & & & \\
\hline \multirow{3}{*}{$\begin{array}{r}\text { INTERVENTION: } \\
\text { CT+Memory Support } \\
\text { CT-as-usual }\end{array}$} & & & & & & & \\
\hline & & & & $X$ & & & \\
\hline & & & & $X$ & & & \\
\hline \multicolumn{8}{|l|}{$\begin{array}{r}\text { DATA } \\
\text { COLLECTION: }\end{array}$} \\
\hline \multirow{4}{*}{$\begin{array}{r}\text { Demographics } \\
\text { Medical History } \\
\text { Diagnostic } \\
\text { Symptomatic }\end{array}$} & & $x$ & & & $X$ & $\mathrm{X}$ & $X$ \\
\hline & & $X$ & & & & & \\
\hline & & $\mathrm{X}$ & & & $\mathrm{X}$ & $\mathrm{X}$ & $\mathrm{X}$ \\
\hline & & $X$ & & $x$ & $\mathrm{X}$ & $X$ & $x$ \\
\hline \multirow{2}{*}{ Functional Impairment } & & $X$ & & & $\mathrm{X}$ & $\mathrm{X}$ & $X$ \\
\hline & & $\bar{X}$ & & & & & \\
\hline \multirow{5}{*}{$\begin{array}{r}\text { Memory Assessment } \\
\text { MSRS } \\
\text { Medication and Other } \\
\text { Treatment Tracking } \\
\text { Credibility/Expectancy } \\
\text { Exploratory Measures }\end{array}$} & & $X$ & & $X$ & $X$ & $x$ & $X$ \\
\hline & & & & $\mathrm{X}$ & & & \\
\hline & & $x$ & & $x$ & $x$ & $x$ & $x$ \\
\hline & & & & $X$ & $\mathrm{X}$ & $X$ & $x$ \\
\hline & & $x$ & & $x$ & $\mathrm{x}$ & $\mathrm{X}$ & $x$ \\
\hline
\end{tabular}

Fig. 2 Standard Protocol Items: SPIRIT Figure: Schedule of enrollment, interventions, and assessments

will be administered along with the Longitudinal Interval Follow-up Evaluation (LIFE) [57] to further ascertain presence/absence of MDD, number and length of mood episodes and remission, response, relapse, recovery, recurrence and time to relapse or recurrence (see Additional file 1 for definitions which are drawn from the ACNP criteria [58]).

\section{Symptomatic}

The IDS-SR [59], a widely used self-report measure of depression severity, as well as two subscales (ideation and behavior) from the lifetime and current version of the Columbia-Suicide Severity Rating Scale (C-SSRS) [60-62], will be administered. The Quick Inventory for Depression Symptomatology (QIDS) is administered every treatment session [38] for clinical purposes (monitoring of suicide and depression symptoms).

\section{Functional impairment}

The World Health Organization Disability Assessment Schedule 2.0 (WHODAS 2.0) [39] as well as the fourquestion "Healthy Days" (CDC HRQOL-4) core module developed by the CDC [63] will be administered.

\section{IQ}

The National Adult Reading Test (NART) [64] estimates premorbid intelligence. The total number of errors are calculated and used to derive an estimate of full-scale IQ and verbal IQ.

\section{Memory}

The Patient Treatment Recall Task [10] and the Generalization Task [65] are included as early indicators of target engagement and the trajectory of recall and learning over time. Classical tests of memory include a test of declarative memory-the Episodic Face-Name Learning Task [66-68]-which is the domain in which the most profound declines in MDD are observed [69-74]. Working memory will be assessed via the $N$-Back [75] because working memory aids the formation and retention of long-term declarative memories via attentive and elaborative-rehearsal processes [76, 77].

\section{Memory Support Rating Scale (MSRS) [78]}

Selected treatment session video-recordings will be coded using the MSRS to establish the dose of memory support delivered.

Medication and other treatment tracking logs will be used to record the additional treatments patients are 
receiving and any changes in those treatments throughout the duration of the study.

Treatment credibility/expectancy is administered at session 2, post-treatment, $6 \mathrm{FU}$ and $12 \mathrm{FU}$ via the Credibility/Expectancy Questionnaire [79, 80].

\section{Exploratory measures}

As this is a relatively new program of research and we wish to learn as much as possible, we have included the following measures on a pilot/exploratory basis: Patient Usefulness and Utilization of Cognitive Therapy Skills Scale (delivered post treatment, 6FU and 12FU), the Competencies in Cognitive Therapy Scale [81] (delivered at post treatment, 6FU and 12FU), Patient Conceptualization of Depression Task (delivered at the pre-treatment assessment, post treatment and 6FU) and a Memory Support Treatment Provider Checklist to be completed by the therapists delivering CT + Memory Support (delivered during weeks 4, 8, 12 and 16 of the treatment).

\section{Treatments}

Both treatments are comprised of 20-26 $\times 50-\mathrm{min}$ sessions conducted over 16 weeks.

\section{CT-as-usual}

CT was developed by Beck et al. [82] and has incorporated a number of innovations [83, 84]. Treatment maneuvers are designed to identify, reality test, and correct distorted beliefs and information processing [82]. CT for MDD will be conducted according to the standard manuals $[82,85,86]$.

\section{$C T+$ Memory Support}

The Memory Support Intervention is a manualized adjunctive treatment that will be delivered alongside CTas-usual. The Memory Support Intervention is designed to improve patient memory for treatment. Distilled from the cognitive science and education literature based on carefully honed criteria [18], the Memory Support Intervention is comprised of eight memory-promoting strategies: attention recruitment, categorization, evaluation, application, repetition, practice remembering, cue-based reminder and praise recall. These strategies are proactively, strategically and intensively integrated into treatment-as-usual to support encoding. Memory support is delivered alongside each "treatment point," defined as a main idea, principle, or experience that the treatment provider wants the patient to remember or implement as part of the treatment [10]. We acknowledge that some memory support is a standard part of certain treatments, including CBT $[84,87]$. In the present study, the two treatment arms will differ in the "dose" of memory support. An underpowered pilot study indicated that the Memory Support Intervention effectively increases the amount of memory support delivered and improves depression outcome on certain measures [34].

\section{Intervention fidelity}

Treatment session video-recordings that occur at session 2 and at weeks 4, 8, 12 and 16 are coded using the MSRS [78] to establish the dose of memory support delivered. CT + Memory Support therapists are blind to which sessions are coded. CT-as-usual therapists are blind to the memory support portion of the research.

To reduce expectations impacting the delivery of each arm and to ensure purity of delivery [88], each treatment provider will be allocated to deliver only one of the two treatments. The rationale is that therapists report that once they have mastered memory support it is difficult to deliver CT-as-usual.

Clinicians use a treatment manual and receive weekly supervision to standardize treatment administration. Treatment sessions are video- and audio-taped and a random selection are rated using the Cognitive Therapy Rating Scale [89] which is a measure of general intervention skills and CBT-specific skills. The goal of this intervention is to maintain consistency and ensure adherence to the protocols [89].

\section{Data analysis \\ Preliminary data evaluation}

Missing or aberrant data will be verified. Data will be audited for quality and completeness. Evaluation of distributions detects outliers and ensures that assumptions of planned analyses are met. Baseline differences between groups will be examined (e.g., IQ, demographics, psychiatric and medical comorbidity, medications). Statistical tests will not be used to select covariates in the primary intent-to-treat analysis [90-92]. Instead, the potential influences of baseline differences will be evaluated as moderators. If remedial training is given to a therapist whose adherence to the treatment protocol is not satisfactory, this will be included as a dummy-coded covariate in all analyses.

\section{Multiple testing}

We will use alpha $=.05$ for each primary hypothesis. For multiple testing conducted within each main hypothesis, we will adopt a stepwise multiple comparison procedure that is considered more powerful than the widely used Bonferroni correction [93, 94].

\section{Missing data}

Longitudinal analyses will use all available data and produce valid inferences if attrition depends on treatment group or on previous outcomes for the same participant [95]. If dropout is associated with other variables, they 
will be added as predictors to reduce bias due to data missing not at random.

\section{Hypothesis 1}

$C T+$ Memory Support will be superior to CT-as-usual for improving course of illness and reducing functional impairment at post-treatment, $6 F U$ and $12 F U$ on primary outcomes listed in Additional file 1. Treatment groups will be compared on categorical outcomes (e.g., remission, relapse) using logistic regression to evaluate the odds of remission at post-treatment, and the odds of relapse at $6 \mathrm{FU}$ and $12 \mathrm{FU}$. For the continuous variables (e.g., IDS-SR), we will test for differences in the mean trajectories across time between CT + Memory Support and CT-as-usual using Hierarchical Linear Modeling (HLM) [96-98]. The 1st level will represent withinperson variation, and will include time indicators (or dummy variables) as predictors (post-treatment, 6FU and 12FU, with baseline as reference). The 2nd level represents between-person variation in the intercept and coefficients of the time indicators, and will include a dummy variable for arm (CT + Memory Support vs. CTas-usual) as the predictor variable. Interactions between arm and the time indicators will be retained only if found to be significant at the $5 \%$ level using likelihoodratio tests. Significant interaction terms between arm and time indicator suggest that there are different trajectories across time and between arms, and will be graphed to interpret the interaction.

\section{Hypothesis 2}

Relative to CT-as-usual, the relationship between CT + Memory Support and better treatment outcome will be mediated by patient memory for treatment. Outcome will be measured by IDS-SR at post-treatment, 6FU and 12FU. Patient memory will be measured by the Patient Treatment Recall Task and the Generalization Task at weeks 4, 8, 12 and 16 of treatment. A mediation model will be specified using Structural Equation Modeling [99]. Indirect effects from CT + Memory Support to better treatment outcome via patient memory will be assessed.

\section{Hypothesis 3}

The relationship between treatment condition and outcome will be moderated by poorer response subgroups; namely, older age, lower IQ, more chronic depression, poorer baseline declarative memory performance and fewer years of education. IQ will be estimated with the NART. Chronicity will be defined as current episode greater than or equal to 2 years [46]. Declarative memory will be quantified as described in Additional file 1. To assess whether Memory Support is more beneficial for any of the patient subgroups, moderation will be tested as an interaction between potential moderator and treatment arm; in the HLMs, the interaction between any of these variables, time, and treatment group will be tested.

\section{Intervention fidelity check}

MSRS scores will be compared for CT + Memory Support vs. CT-as-usual via independent samples $t$ tests. To test the assumption that memory for CT session content improves as a result of $\mathrm{CT}+$ Memory Support we will use HLMs to analyze differences in linear rates of change between the two groups on the Patient Treatment Recall Task and Generalization Task delivered during weeks 4, 8, 12 and 16 of the treatment phase and at $6 \mathrm{FU}$ and $12 \mathrm{FU}$.

\section{Additional planned analyses}

1. Is CT + Memory Support associated with longer timeto-relapse relative to CT-as-usual? Survival analyses [100] will be conducted. The Kaplan-Meier product limit method will be used to generate survival curves for the two treatment conditions [101]. Cox regression will be applied to test for group differences.

2. Are demographics, psychiatric and medical comorbidity, medications and symptom severity moderators? This will be tested by adding the product of arm and the moderator variable to regression models or HLMs [102, 103]. A significant coefficient for the interaction term would indicate a moderating effect, and will be followed up with graphs to determine the nature of the effect modification [102].

3. Does memory for treatment improve simply because memory deficits resolve with successful treatment? Linear regressions will evaluate whether change in pre-post IDS and pre-post declarative and working memory each independently predict week 4 to posttreatment differences in Patient Recall Task performance. If significant, we will conduct a hierarchical linear regression to evaluate whether pre-post change in IDS scores predicts pre-post change in Patient Treatment Recall Task performance beyond pre-post improvement in memory.

4. Memory Support Rating Scale data will be used to delineate the most effective types and combinations of memory support. Factor analysis will be used to test the structure of the Memory Support Rating Scale based on Lee et al. [78]. Associations between patient recall, depression outcomes, and memory support variables will be assessed. 
5. Number of sessions received (range 20-26 sessions) will be added as a covariate to control for differences in this across participants.

\section{Power analysis}

Based on the pilot study [34], using Glimmpse [104, 105] for repeated measures design, we took into account the four repeated measurements (pre-treatment, posttreatment, 6FU, 12FU). At an alpha level of 0.05 with $80 \%$ power, the sample size is estimated to be 80 for the IDS-SR. We also used G*Power and the Demidenko [106] method to calculate estimated sample size for binary mood outcomes (i.e., remission, relapse). The sample sizes are estimated to be 103 (remission) and 118 (relapse). Finally, Fritz and MacKinnon [107] provide recommendations for mediation sample size, and estimates from pilot data indicate that the recommended sample size is 148 . Adding an additional $20 \%$ for possible attrition, we propose a sample of 178 .

\section{Discussion}

This study protocol addresses several research priorities. First, following the experimental therapeutics approach [108], this study protocol describes a confirmatory efficacy trial to provide a definitive test of the hypothesis that a novel target-patient memory for the contents of treatment-probed with a novel intervention-the Memory Support Intervention-will improve clinical outcome. Second, a novel intervention derived from basic, non-patient research in cognitive science and education, will be tested in the service of improving outcomes for patients with severe mental disorders. Third, the Memory Support Intervention has been derived to be "transdiagnostic" (relevant to a broad range of mental disorders) and "pantreatment" (relevant to a broad range of types of treatment). However, investigating these possible broader applications seems premature without first establishing efficacy, which is the aim of the study described in this protocol. If the results are promising, future directions will test the applicability to a broad range of biopsychosocial interventions for a wide range of patient populations being treated at various medical and mental health settings.

\section{Trial status}

The trial is funded for 4 years (Project ID Number: R01MH108657). The research staff team started setting up the study in September, 2016. Patients were first randomized in December, 2016. The treatment phase will be completed by August of 2019. Final outcome assessments will be complete by August of 2020 .

\section{Endnotes}

${ }^{1}$ This was added in July, 2017. NIH/NIMH datasharing requirements necessitated reconsent of participants already randomized. If a patient does not agree to data sharing we have been instructed to exclude them from the analysis.

\section{Additional files}

Additional file 1: SPIRIT 2013 Checklist. (DOC 122 kb)

Additional file 2: Summary of primary and secondary outcome(s). (DOCX $13 \mathrm{~kb}$ )

Additional file 3: Timeframe for Assessments. Table indicating time points where assessments are conducted. (DOCX $46 \mathrm{~kb}$ )

\section{Abbreviations}

12FU: 12-months' follow-up assessment; 6FU: 6-months' follow-up assessment; ACNP: American College of Neuropsychopharmacology; CBT: Cognitive behavior therapy; CEQ: Credibility/Expectancy Questionnaire: C-SSRS: Columbia-Suicide Severity Rating Scale; CT: Cognitive therapy; CTRS: Cognitive Therapy Rating Scale; DSM-5: Diagnostic and Statistical Manual of Mental Disorders, Fifth Edition; DSMB: Data and Safety Monitoring Board; HLM: Hierarchical Linear Modeling; IDS-SR: Inventory of Depressive Symptoms, Self-Report; LIFE: Longitudinal Interval Follow-up Evaluation; MDD: Major depressive disorder; MSRS: Memory Support Rating Scale; NART: National Adult Reading Test; NIMH: National Institutes of Mental Health; QIDS: Quick Inventory of Depressive Symptoms; SEM: Structural Equation Modeling; SPIRIT 2013: Standard Protocol Items: Recommendations for Interventional Trials; WHODAS 2.0: The World Health Organization Disability Assessment Schedule 2.0

\section{Acknowledgements}

This study is funded by the National Institute of Mental Health (R01MH108657). The DSMB is composed of three members: Dr. John McQuaid, PhD, Dr. Jutta Joormann, PhD and Dr. Hao Wu.

The DSMB reviews progress and safety of study procedures twice per year and are responsible for safe guarding the interests of study participants. This committee is independent of the sponsor.

\section{Funding}

This study is funded by the National Institute of Mental Health (R01MH108657). The funding agency has/had no role in the design, collection, management, analysis, or interpretation of data; the writing of the manuscript; or the decision to submit the study protocol for publication. The funding agency has no ultimate authority over any of these activities.

\section{Availability of data and materials}

Not applicable

\section{Authors' contributions}

AGH developed the study concept. AGH, LD, JL, SH and S R-H contributed to the acquisition of the funding. $A G H$ and $J$ contributed to developing the Memory Support Intervention. SDH, $\mathrm{KHa}$ and $\mathrm{AGH}$ trained the therapists. $A G H, N B G, K H e, M M, C W, H N, A M, G Z$ and $C A$ are responsible for the acquisition of data and the measurement of memory. LD, AM and S R-H are responsible for the analysis and interpretation of data. AGH drafted the manuscript. All authors have been involved in revising the manuscript. All authors read and approved the final manuscript. Other than the authors and compliance with data-sharing agreements stipulated by $\mathrm{NIH}$, no other entities have contractual agreements with regard to access to the final dataset.

\section{Ethics approval and consent to participate}

The University of California, Berkeley, Committee for the Protection of Human Subjects (CPHS) approved the study (Protocol ID: 2011-11-3795). As depicted in Fig. 2, verbal informed consent is obtained during the initial eligibility assessment which is typically conducted over the telephone. This is 
followed by a written informed consent which is obtained at the beginning of the pre-assessment, which confirms eligibility, by a member of the assessment team. Adverse events and other unintended effects will be report to $\mathrm{CPHS}$ and $\mathrm{NIH}$, following the rules stipulated by these two oversight bodies. If important protocol modifications are made these will be reviewed by CPHS and reported on ClinicalTrials.gov.

\section{Consent for publication}

\section{Not applicable}

\section{Competing interests}

The authors declare that they have no competing interests.

\section{Publisher's Note}

Springer Nature remains neutral with regard to jurisdictional claims in published maps and institutional affiliations.

\section{Author details}

'Department of Psychology, University of California, Berkeley, 3210 Tolman Hall \#1650, Berkeley, CA 94720-1650, USA. Vanderbilt University, Nashville, TN, USA.

\section{Received: 25 August 2017 Accepted: 23 October 2017}

Published online: 14 November 2017

\section{References}

1. Bober SL, Hoke LA, Duda RB, Tung NM. Recommendation recall and satisfaction after attending breast/ovarian cancer risk counseling. J Genet Couns. 2007;16:755-62

2. Jansen J, Butow PN, van Weert JC, van Dulmen S, Devine RJ, Heeren TJ, et al. Does age really matter? Recall of information presented to newly referred patients with cancer. J Clin Oncol. 2008;26:5450-7.

3. Pickney CS, Arnason JA. Correlation between patient recall of bone densitometry results and subsequent treatment adherence. Osteoporos Int. 2005:16:1156-60.

4. Lewkovich GN, Haneline MT. Patient recall of the mechanics of cervical spine manipulation. J Manipulative Physiol Ther. 2005;28:708-12.

5. Croyle RT, Loftus EF, Barger SD, Sun YC, Hart M, Gettig J. How well do people recall risk factor test results? Accuracy and bias among cholesterol screening participants. Health Psychol. 2006;25:425-32.

6. Mcguire LC. Remembering what the doctor said: organization and adults' memory for medical information. Exp Aging Res. 1996;22(4):403-28.

7. Ley P. Memory for medical information. Br J Soc Clin Psychol. 1979;18: 245-55

8. Jansen J, van Weert J, van der Meulen N, van Dulmen S, Heeren T, Bensing $J$. Recall in older cancer patients: measuring memory for medical information. Gerontologist. 2008;48(2):149-57.

9. Chambers MJ. Patient recall of recommendations in the behavioura treatment of insomnia. Sleep Res. 1991;20:222.

10. Lee J, Harvey AG. Memory for therapy in bipolar disorder and comorbid insomnia. J Consult Clin Psychol. 2015;83:92-102. PubMed Central PMCID: PMCPMC4323885.

11. Flocke SA, Stange KC. Direct observation and patient recall of health behavior advice. Prev Med. 2004;38:343-9.

12. Tosteson AN, Grove MR, Hammond CS, Moncur MM, Ray GT, Hebert GM, et al. Early discontinuation of treatment for osteoporosis. Am J Med. 2003 115:209-16.

13. Cox DJ, Tisdelle DA, Culbert JP. Increasing adherence to behavioral homework assignments. J Behav Med. 1988;11(5):519-22.

14. Linn AJ, van Dijk L, Smit EG, Jansen J, van Weert JC. May you never forget what is worth remembering: the relation between recall of medical information and medication adherence in patients with inflammatory bowel disease. J Crohn's Colitis. 2013;7(11):e543-50.

15. Ley $P$, Jain $V$, Skilbeck C. A method for decreasing patients' medication errors. Psychol Med. 1977;6(04):599-601.

16. Kravitz RL, Hays RD, Sherbourne CD, DiMatteo MR, Rogers WH, Ordway L, et al. Recall of recommendations and adherence to advice among patients with chronic medical conditions. Arch Intern Med. 1993:153:1869-78.

17. Simpson HB, Maher MJ, Wang Y, Bao Y, Foa EB, Franklin M. Patient adherence predicts outcome from cognitive behavioral therapy in obsessive-compulsive disorder. J Consult Clin Psychol. 2011;79(2):247.
18. Harvey AG, Lee J, Williams J, Hollon SD, Walker MP, Thompson MA, et al. Improving outcome of psychosocial treatments by enhancing memory and learning. Perspect Psychol Sci. 2014;9:161-79. PubMed Central PMCID: PMCPMC4276345.

19. Schacter $D$. The seven sins of memory: how the mind forgets and remembers. New York City: Houghton Mifflin; 2001.

20. Easterbrook JA. The effect of emotion on cue utilization and the organization of behavior. Psychol Rev. 1959;66:183-201. PubMed PMID: 1961-03074-001.

21. Thorndike EL. The fundamentals of learning. New York: Teacher's College Bureau of Publications; 1932

22. Barnett SM, Ceci SJ. When and where do we apply what we learn? A taxonomy for far transfer. Psychol Bull. 2002;128:612.

23. Behnken A, Schöning S, Gerss J, Konrad C, de Jong-Meyer R, Zwanzger P, et al. Persistent non-verbal memory impairment in remitted major depression_caused by encoding deficits? J Affect Disord. 2010;122:144-8.

24. Robinson $\amalg$, Thompson JM, Gallagher P, Goswami U, Young AH, Ferrier IN, et al. A meta-analysis of cognitive deficits in euthymic patients with bipolar disorder. J Affect Disord. 2006;93:105-15.

25. Varga M, Magnusson A, Flekkoy K, David AS, Opjordsmoen S. Clinical and neuropsychological correlates of insight in schizophrenia and bipolar I disorder: does diagnosis matter? Compr Psychiatry. 2007:48:583-91.

26. Jelinek L, Jacobsen D, Kellner M, Larbig F, Biesold K-H, Barre K, et al. Verbal and nonverbal memory functioning in posttraumatic stress disorder (PTSD). J Clin Exp Neuropsychol. 2006;28:940-8.

27. Airaksinen $E$, Larsson M, Forsell Y. Neuropsychological functions in anxiety disorders in population-based samples: evidence of episodic memory dysfunction. J Psychiatr Res. 2005;39:207-14.

28. Bruce JM, Hancock LM, Arnett P, Lynch S. Treatment adherence in multiple sclerosis: association with emotional status, personality, and cognition. J Behav Med. 2010:33(3):219-27.

29. Insel K, Morrow D, Brewer B, Figueredo A. Executive function, working memory, and medication adherence among older adults. J Gerontol B Psychol Sci Soc Sci. 2006;61(2):102-7.

30. Ley P, Bradshaw P, Eaves D, Walker C. A method for increasing patients' recall of information presented by doctors. Psychol Med. 1973;3:217-20.

31. Bamidis PD, Vivas AB, Styliadis C, Frantzidis C, Klados M, Schlee W, Siountas A, Papageorgiou SG. A review of physical and cognitive interventions in aging. Neurosci Biobehav Rev. 2014;44:206-20.

32. Almkvist O, Fratiglioni L, Agüero-Torres H, Viitanen M, Bäckman L. Cognitive support at episodic encoding and retrieval: similar patterns of utilization in community-based samples of Alzheimer's disease and vascular dementia patients. J Clin Exp Neuropsychol. 2010;21:816-30.

33. Bunce D. Cognitive support at encoding attenuates age differences in recollective experience among adults of lower frontal lobe function. Neuropsychology. 2003;17:353-61.

34. Harvey AG, Lee J, Smith RL, Gumport NB, Hollon SD, Rabe-Hesketh S, et al Improving outcome for mental disorders by enhancing memory for treatment. Behav Res Ther. 2016;81:35-46.

35. Lopez AD, Mathers CD. Measuring the global burden of disease and epidemiological transitions: 2002-2030. Ann Trop Med Parasitol. 2006; 100(5-6):481-99.

36. Mathers CD, Loncar D. Projections of global mortality and burden of disease from 2002 to 2030. PLoS Med. 2006:3(11):e442.

37. Eaton WW, Shao H, Nestadt G, Lee BH, Bienvenu OJ, Zandi P. Populationbased study of first onset and chronicity in major depressive disorder. Arch Gen Psychiatry. 2008;65(5):513-20.

38. Rush A, Trivedi M, Ibrahim H, Carmody T, Arnow B, Klein D, et al. The 16item Quick Inventory of Depressive Symptomatology (QIDS) Clinician Rating (QIDS-C) and Self-Report (QIDS-SR): a psychometric evaluation in patients with chronic major depression. Biol Psychiatry. 2003;54:573-83.

39. World Health Organization. 2.0: WHODAS 2.0. 2011.

40. Dong L, Lee JY, Harvey AG. Memory support strategies and bundles: a pathway to improving cognitive therapy for depression? J Consult Clin Psychol. 2017:85(3):187.

41. Dong L, Zhao X, Ong SL, Harvey AG. Patient recall of specific cognitive therapy contents predicts adherence and outcome. Behav Res Ther. 2017; 97:189-99.

42. Cuijpers $P$, Hollon SD, van Straten A, Bockting C, Berking M, Andersson G. Does cognitive behaviour therapy have an enduring effect that is superior to keeping patients on continuation pharmacotherapy? A meta-analysis. $\mathrm{Br}$ Med J Open. 2013;3:4 
43. Cuijpers P, Berking M, Andersson G, Quigley L, Kleiboer A, Dobson KS. A meta-analysis of cognitive-behavioural therapy for adult depression, alone and in comparison with other treatments. Can J Psychiatry. 2013;58:7.

44. Bockting CL, Hollon SD, Jarrett RB, Kuyken W, Dobson K. A lifetime approach to major depressive disorder: the contributions of psychological interventions in preventing relapse and recurrence. Clin Psychol Rev. 2015 41:16-26.

45. Jarrett RB, Minhajuddin A, Kangas JL, Friedman ES, Callan JA, Thase ME. Acute phase cognitive therapy for recurrent major depressive disorder: who drops out and how much do patient skills influence response? Behav Res Ther. 2013;51(4):221-30.

46. Fournier JC, DeRubeis RJ, Shelton RC, Hollon SD, Amsterdam JD, Gallop R. Prediction of response to medication and cognitive therapy in the treatment of moderate to severe depression. J Consult Clin Psychol. 2009;77(4):775.

47. Majer M, Ising M, Künzel H, Binder EB, Holsboer F, Modell S, et al. Impaired divided attention predicts delayed response and risk to relapse in subjects with depressive disorders. Psychol Med. 2004;34:1453-63.

48. Bearden CE, Glahn DC, Monkul ES, Barrett J, Najt P, Villarreal V, et al. Patterns of memory impairment in bipolar disorder and unipolar major depression. Psychiatry Res. 2006;142(2):139-50.

49. Deuschle M, Kniest A, Niemann H, Erb-Bies N, Colla M, Hamann B, et al. Impaired declarative memory in depressed patients is slow to recover: clinical experience. Pharmacopsychiatry. 2004;37(04):147-51.

50. Bremner JD, Vythilingam $M$, Vermetten E, Vaccarino V, Charney DS Deficits in hippocampal and anterior cingulate functioning during verbal declarative memory encoding in midlife major depression. Am J Psychiatry. 2004;161(4):637-45.

51. Douglas KM, Porter RJ. Longitudinal assessment of neuropsychological function in major depression. Aust N Z J Psychiatry. 2009;43(12):1105-17.

52. Chan A-W, Tetzlaff JM, Gøtzsche PC, Altman DG, Mann H, Berlin JA, et al. SPIRIT 2013 explanation and elaboration: guidance for protocols of clinical trials. BMJ. 2013;346:e7586.

53. Daumit GL, Dickerson FB, Wang N-Y, Dalcin A, Jerome GJ, Anderson CA, et al. A behavioral weight-loss intervention in persons with serious mental illness. N Engl J Med. 2013;368(17):1594-602.

54. McGrath CL, Kelley ME, Dunlop BW, Holtzheimer III PE, Craighead WE, Mayberg HS. Pretreatment brain states identify likely nonresponse to standard treatments for depression. Biol Psychiatry. 2014;76(7):527-35.

55. First MB, Spitzer RL, Gibbon M, Williams JBW. Structured clinical interview for DSM-V Axis 1 disorders administration booklet. New York: American Psychiatric Press; 2013.

56. First MB, Williams JBW, Karg RS, Spitzer RL. Structured Clinical Interview for DSM-5 Disorders (SCID-5-RV, FEB 2014 revision). New York: Biometrics Research Department, Columbia University; 2014.

57. Keller MB, Lavori PW, Friedman B, Nielsen E, Endicott J, McDonald S, et al. The longitudinal interval follow-up evaluation: a comprehensive method for assessing outcome in prospective longitudinal studies. Arch Gen Psychiatry. 1987:44:540-8. PubMed PMID: 1988-01549-001.

58. Rush AJ, Kraemer HC, Sackeim HA, Fava M, Trivedi MH, Frank E, et al. Report by the ACNP Task Force on response and remission in major depressive disorder. Neuropsychopharmacology. 2006;31(9):1841-53.

59. Trivedi MH, Rush AJ, Ibrahim HM, Carmody TJ, Biggs MM, Suppes T, et al. The Inventory of Depressive Symptomatology, Clinician Rating (IDS-C) and Self-Report (IDS-SR), and the Quick Inventory of Depressive Symptomatology, Clinician Rating (QIDS-C) and Self-Report (QIDS-SR) in public sector patients with mood disorders: a psychometric evaluation. Psychol Med. 2004;34:73-82.

60. Center for Drug Evaluation and Research. Guidance for industry: suicidal ideation and behavior: prospective assessment of occurrence in clinical trials. Rockville: U.S. Department of Health and Human Services, U.S. Food and Drug Administration; 2012.

61. Mundt JC, Greist JH, Jefferson JW, Federico M, Mann JJ, Posner K. Prediction of suicidal behavior in clinical research by lifetime suicidal ideation and behavior ascertained by the electronic Columbia-Suicide Severity Rating Scale. J Clin Psychiatry. 2013;74(9):887-93.

62. Posner K, Brown GK, Stanley B, Brent DA, Yershova KV, Oquendo MA, Currier GW, Melvin GA, Greenhill L, Shen S, Mann JJ. The Columbia-Suicide Severity Rating Scale: initial validity and internal consistency findings from three multisite studies with adolescents and adults. Am J Psychiatry. 2011;168(12): 1266-77.
63. Moriarty D, Zack M, Kobau R. The Centers for Disease Control and Prevention's Healthy Days Measures: population tracking of perceived physical and mental health over time. Health Qual Life Outcomes. 2003;1(1):37.

64. Nelson HE, Willison J. National Adult Reading Test (NART). Windsor: NFERNelson; 1991.

65. Gumport NB, Williams JJ, Harvey AG. Learning cognitive behavior therapy. J Behav Ther Exp Psychiatry. 2015;48:164-9. PubMed Central PMCID: PMCPMC4426215.

66. Mander BA, Santhanam S, Saletin JM, Walker MP. Wake deterioration and sleep restoration of human learning. Curr Biol. 2011;21:R183-4.

67. Miller SL, Celone K, DePeau K, Diamond E, Dickerson BC, Rentz D, et al. Age-related memory impairment associated with loss of parietal deactivation but preserved hippocampal activation. Proc Natl Acad Sci. 2008;105(6):2181-6.

68. Sperling R, Bates J, Chua E, Cocchiarella A, Rentz D, Rosen B, et al. fMRI studies of associative encoding in young and elderly controls and mild Alzheimer's disease. J Neurol Neurosurg Psychiatry. 2003;74(1):44-50.

69. Taconnat L, Baudouin A, Fay S, Raz N, Bouazzaoui B, El-Hage W, et al. Episodic memory and organizational strategy in free recall in unipolar depression: the role of cognitive support and executive functions. J Clin Exp Neuropsychol. 2010;32:719-27.

70. Danion JM, Willard-Schroeder D, Zimmermann MA, Grangé D, Schlienger JL, Singer L. Explicit memory and repetition priming in depression. Preliminary findings Arch Gen Psychiatry. 1991;48:707-11.

71. Jermann F, Van der Linden M, Adam S, Ceschi G, Perroud A. Controlled and automatic uses of memory in depressed patients: effect of retention interval lengths. Behav Res Ther. 2005;43:681-90.

72. Calev A, Erwin PG. Recall and recognition in depressives: use of matched tasks. Br J Clin Psychol. 1985;24:127-8.

73. Ellwart $T$, Rinck $M$, Becker ES. Selective memory and memory deficits in depressed inpatients. Depress Anxiety. 2003;17:197-206.

74. Hertel PT, Milan S. Depressive deficits in recognition: dissociation of recollection and familiarity. J Abnorm Psychol. 1994;103:736-42.

75. Kirchner WK. Age differences in short-term retention of rapidly changing information. J Exp Psychol. 1956;55:352-8.

76. Cowan N. What are the differences between long-term, short-term, and working memory? Prog Brain Res. 2008;169:323-38.

77. Ericsson KA, Kintsch W. Long-term working memory. Psychol Rev. 1995;102(2):211.

78. Lee JY, Worrell FC, Harvey AG. The Development and Validation of the Memory Support Rating Scale (MSRS). Psychol Assess. 2016;28(6):715-25

79. Devilly GJ, Borkovec TD. Psychometric properties of the credibility/ expectancy questionnaire. J Behav Ther Exp Psychiatry. 2000;31:73-86. PubMed PMID: 2000-12660-001.

80. Devilly GJ, Spence SH. The relative efficacy and treatment distress of EMDR and a cognitive-behavior trauma treatment protocol in the amelioration of posttraumatic stress disorder. J Anxiety Disord. 1999;13:131-57. PubMed PMID: 1999-13238-007.

81. Strunk DR, Hollars SN, Adler AD, Goldstein LA, Braun JD. Assessing patients' cognitive therapy skills: initial evaluation of the competencies of Cognitive Therapy Scale. Cogn Ther Res. 2014;38(5):559.

82. Beck AT. Cognitive therapy of depression. New York: Guilford Press; 1979. p. 425.

83. Bennett-Levy J, Butler G, Fennell MJV, Hackmann A, Mueller M, Westbrook D. The Oxford handbook of behavioural experiments. Oxford: Oxford University Press; 2004.

84. Beck JS. Cognitive therapy: basics and beyond. New York: Guilford Press; 1995.

85. Beck JS. Cognitive therapy: basics and beyond. 2nd ed. New York: Guilford Press; 2011.

86. Greenberger D, Padesky CA. Mind over mood: change how you feel by changing the way you think. New York: Guilford Press; 2015.

87. Beck JS. Cognitive therapy for challenging problems: what to do when the basics don't work. New York: Guilford Press; 2005. p. 324

88. Manber R, Edinger JD, Gress JL, San Pedro-Salcedo MG, Kuo TF, Kalista T. Cognitive behavioral therapy for insomnia enhances depression outcome in patients with comorbid major depressive disorder and insomnia. Sleep. 2008:31:489-95.

89. Young J, Beck AT. The development of the Cognitive Therapy Scale. 1980.

90. Beach ML, Meier P. Choosing covariates in the analysis of clinical trials. Control Clin Trials. 1989;10:161S-75. 
91. Canner PL. Covariate adjustment of treatment effects in clinical trials. Control Clin Trials. 1991;12:359-66.

92. Senn SJ. Covariate imbalance and random allocation in clinical trials. Stat Med. 1989:8:467-75.

93. Nakagawa S. A farewell to Bonferroni: the problems of low statistical power and publication bias. Behav Ecol. 2004;15:1044-5. PubMed PMID: 200419626-022.

94. Shaffer JP. Multiple hypothesis testing. Annu Rev Psychol. 1995;46(1):561-84.

95. Little RJ, Rubin DB. Statistical analysis with missing data. 2nd ed. New York: Wiley; 2002.

96. Raudenbush S, Bryk A. Hierarchical linear models. Thousand Oaks: Sage; 2002.

97. Goldstein H. Multilevel statistical models. 4th ed. Chichester: Wiley; 2010

98. Hox J. Multilevel analysis: techniques and applications. NY: Routledge; 2010.

99. Hayes AF. Introduction to mediation, moderation, and conditional process analysis: a regression-based approach. New York: Guilford Press; 2013.

100. Greenhouse JB, Stangl D, Bromberg J. An introduction to survival analysis: statistical methods for analysis of clinical trial data. J Consult Clin Psychol. 1989;57:536-44.

101. Kaplan E, Meier P. Nonparametric estimation from incomplete observations. J Am Stat Assoc. 1958;53:457-81.

102. Cohen J, Cohen P, West S, Aiken L. Applied multiple regression/correlation analysis for the behavioral sciences. 3rd ed. New Jersey: Lawrence Erlbaum Associates; 2003.

103. Jaccard J, Wan CK, Turrisi R. The detection and interpretation of interaction effects between continuous variables in multiple regression. Multivar Behav Res. 1990;25(4):467-78.

104. Guo Y, Logan HL, Glueck DH, Muller KE. Selecting a sample size for studies with repeated measures. BMC Med Res Methodol. 2013;13(1):100.

105. Kreidler SM, Muller KE, Grunwald GK, Ringham BM, Coker-Dukowitz ZT, Sakhadeo UR, et al. GLIMMPSE: online power computation for linear models with and without a baseline covariate. J Stat Softw. 2013;54:10.

106. Demidenko E. Sample size determination for logistic regression revisited. Stat Med. 2007;26(18):3385-97.

107. Fritz MS, Mackinnon DP. Required sample size to detect the mediated effect. Psychol Sci. 2007;18(3):233-9.

108. Insel TR, Gogtay N. National Institute of Mental Health clinical trials: new opportunities, new expectations. JAMA Psychiat. 2014;71(7):745-6.

\section{Submit your next manuscript to BioMed Central and we will help you at every step:}

- We accept pre-submission inquiries

- Our selector tool helps you to find the most relevant journal

- We provide round the clock customer support

- Convenient online submission

- Thorough peer review

- Inclusion in PubMed and all major indexing services

- Maximum visibility for your research

Submit your manuscript at www.biomedcentral.com/submit

) Biomed Central 